\title{
Percutaneous needle electrolysis and exercise reduce the time for return to competition after an injury to the hamstrings: Two cases in professional football players
}

\author{
jiménez Rubio S. ${ }^{1}$ Valera Garrido F. ${ }^{1,2}$ Minaya Muñoz F. ${ }^{2}$ Navandar A. ${ }^{3}$ \\ ${ }^{1}$ Getafe C.F. Spain \\ 2 MVClinic, Madrid, Spain \\ ${ }^{3}$ Universidad Europea de Madrid, Madrid, Spain \\ Rev Fisioter Invasiva 2019;2:121-122.
}

\begin{abstract}
Background and Aims Percutaneous needle electrolysis has shown to be effective for the treatment of chronic tendinopathies, however, the scientific evidence available regarding acute muscle lesions is scarce. Lesions to the hamstrings caused by indirect mechanisms represent the most common muscle lesion in football (its incidence increases each year) with a high rate of recurrence. The aim was to evaluate whether the application of percutaneous needle electrolysis and a program of functional exercise decreases the times for return to competition after an injury to the hamstrings in athletes, and to compare performance data of subjects during competition before and after the injury.

Methods A case series. Two professional football players, belonging to a first league team in Spain with a grade 2 lesion in the semitendinosus muscle on the proximal level. The affected muscle structure was assessed using ultrasound (Logiq GE E9, probe ML6-12) and functionality was evaluated based on data of the Global Positioning System (GPS), comparing the data obtained during the process of re-adaptation with data prior to the lesion. Percutaneous needle electrolysis was performed under ultrasound guidance at 48 hours after the injury following the protocol defined by Valera \& Minaya (2:3:5). The indoor recovery of players began 24 hours after the percutaneous needle electrolysis. Initially, the exercise program included activities of mobility and controlled loading. Thereafter, the players completed their readaptation program in the training field with exercises of progressive complexity and intensity (biomechanical patterns and neuromuscular control of the central zone and the lower limbs, sprint).

Results Player A returned to competition in 16 days, player B returned in 14 days. No adverse effects were identified during or after the percutaneous needle electrolysis technique. The players only missed one competition game and the process of

Keywords

- percutaneous needle electrolysis

- exercise

- return to competition

- football readaptation allowed them to return to play registering parameters which were similar to the values prior to the lesion, and maintaining the performance during the four matches following the intervention protocol. The players were followed up to eight months after the injury and neither player suffered a relapse during this period, which indicates the complete recovery of the injured muscle both on a structural and functional level.
\end{abstract}


Conclusions The combined treatment using percutaneous needle electrolysis and a functional exercise program reduces the time for return to competition after a grade 2 muscle lesion in the hamstrings, with a level of performance that is similar to prior to the lesion, without relapses, in an 8 month follow up period. The criteria we use to determine when a player can return to train with the team and compete after an acute muscle injury are: the time that has passed since the lesion, structural changes and the ability to perform tasks. 